\title{
A Model on Heat Signal of Crystal Detector at Low Temperature
}

\author{
Jin Li · Inwook Kim
}

the date of receipt and acceptance should be inserted later

\begin{abstract}
We present a model to calculate heat signal shapes from low temperature bolometer attached to a crystal. This model is based on the elementary acoustic wave theory at low temperature, and has been developed using modern Monte Carlo techniques. Physical processes in phonon propagation, such as transmission, scattering and reflection are considered. Using our model, the calculated time dependence of signal agrees with real experimental data. This model has applications in low temperature rare event particle detectors for dark matter and neutrinos.
\end{abstract}

Keywords phonon physics, simulation, lattice dynamics, acoustic wave, cryogenic calorimeter

\section{Motivation}

With the development of low temperature detectors for rare event searches, there is a growing interest in understanding observed signals from basic physical principles ${ }^{1 / 2}$. Previous attempts are either limited in time scale or in type of physics processes considered. Thus a new way to quantitatively describe the observables is required.

Here we study signal on solid state crystals, which are widely used to detect energy deposit to it by elementary particles. Phonons are created by initial energy deposit, and then transported to the sensor for detection. The physical principles governing the whole process are known to a large extent ${ }^{3}$. However, it remains to be accomplished to explain and predict the observed signals using those principles.

\section{Physical Processes for Phonon}

In this study, we shall apply our model to experiments where where transmission, scattering and reflection processes all play important roles in the obtained data. We choose detector geometry where the phonons are emitted and detected from two small metal films evaporated

Center for Underground Physics, Institute for Basic Science (IBS), Daejeon, 34047, Korea

Tel.:+82-2-878-8524

E-mail: jinlee@ibs.re.kr 


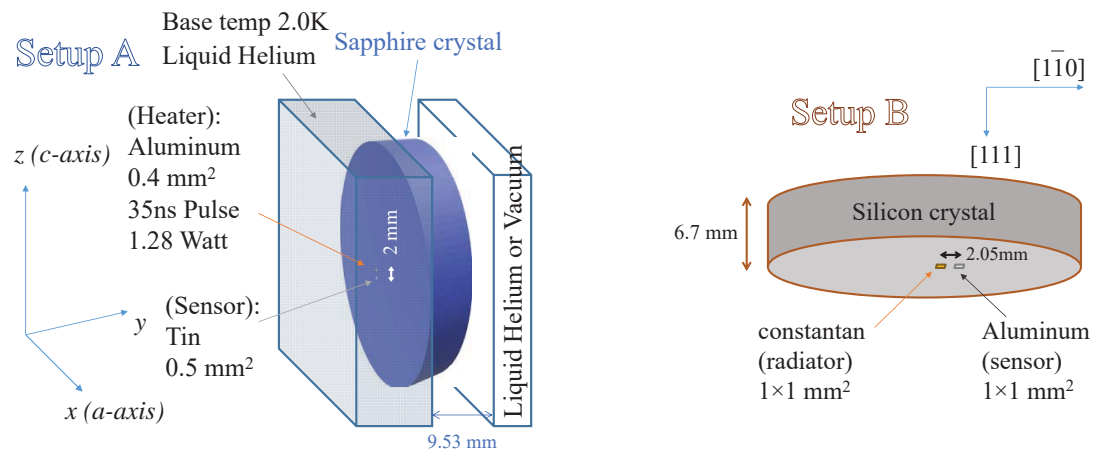

Fig. 1 Experimental setup A (left) and setup B (right). The dimension of the radiator, sensor and crystal substrate are shown.

to the same cut surface of a crystal substrate, such as two setups shown in Fig. 1. Here, in addition to free propagation, the detected phonons must undergo either scattering in the bulk or reflection from the surface of the other side, because the solid angle of a straight line from the radiator source to the sensor is zero. This choice of detector geometry provides a rigorous test to all physical processes considered in our model.

For the detector setups we study in this paper, the phonons are initially created in the radiator which is an ohmic metal heater, then transmit across the interface to the crystal substrate, travel inside it, and are finally absorbed by hitting a superconducting bolometer with metal sensor.

In order to obtain the energy deposit spectrum, we generate individual phonons, and simulate their transportation from initial creation to the final absorption by applying basic physical principles. The physical processes used are discussed below.

\subsection{Phonon emission from the radiator to the crystal substrate}

Initially, phonons are created in the radiator by ohmic heating, and then transmit in to the crystal through the boundary. The metal radiator is normally of polycrystalline nature, which can be described as an isotropic material for acoustic properties. The phonons in the radiator follow the Planck distribution of energy levels. The area of the radiator $A^{(1)}$, the timedependent temperature $T^{(1)}(t)$ of the radiator (1) and base temperature $T^{(0)}$ of crystal (0), are firstly denoted. Then the spectral power of the heat transmission to the crystal $P^{(10)}(\omega, t)$ is 4 .

$P^{(10)}(\omega, t)= \begin{cases}A^{(1)} \frac{\hbar \omega^{3}}{8 \pi^{2}} \sum_{\sigma^{\prime}=1}^{3} \frac{e_{\sigma^{\prime}}^{(10)}}{\left\{c_{\sigma^{\prime}}^{(1)}\right\}^{2}} \cdot\left\{\frac{1}{e^{\hbar \omega / k_{B} T^{(1)}(t)}-1}-\frac{1}{e^{\hbar \omega / k_{B} T^{(0)}}-1}\right\} & \omega<\omega_{\max , \sigma^{\prime}} \\ 0 & \omega>\omega_{\max , \sigma^{\prime}}\end{cases}$

The halfspace emissivities $e_{\sigma^{\prime}}^{(10)}$ from radiator (1) to crystal (0) can be calculated using the acoustic mismatch model as in Ref. ${ }^{5} . c_{\sigma^{\prime}}^{(1)}$ are the isotropic sound velocities for mode $\sigma^{\prime}$ in the radiator. 
In cases discussed in this study, cutoff frequency $\omega_{\max }$ can be considered to be big. By integrating Eq. 1 over frequency $\omega$, the total transmitted power is related to $T^{(1)}(t)$ :

$$
P^{(10)}\left(T^{1}(t), T^{(0)}\right)=A^{(1)} \cdot \frac{\pi^{2} k_{B}^{4}}{120 \hbar^{3}} \sum_{\sigma^{\prime}=1}^{3} \frac{e_{\sigma^{\prime}}^{(10)}}{\left\{c_{\sigma^{\prime}}^{(1)}\right\}^{2}} \cdot\left[\left\{T^{(1)}(t)\right\}^{4}-\left\{T^{(0)}\right\}^{4}\right] .
$$

As an ohmic heat pulse is applied to the radiator, it quickly comes to a steady-state in less than a few ns for a typical thickness of $50 \mathrm{~nm}^{6}$. For the heat pulse longer than tens of ns, we can use a steady-state radiator temperature $T^{(1)}$, calculated by equaling the $P^{(10)}\left(T^{(1)}, T^{(0)}\right)$ to the input electric power.

The differential distribution of the transmitted phonons into the crystal substrate originating from the radiator-crystal interface as a function of the wave vector $\mathbf{q}$ needs to be known to generate phonon directions in half-space. The $\mathbf{q}$-space source distribution is obtained by differential emissivities from acoustic boundary condition. Let the normal of the substrate surface pointing inward be $\mathbf{e}_{3}$, the group velocity of mode $\sigma$ in substrate be $\mathbf{w}_{\sigma}^{(0)}(\mathbf{q})$, the phase velocity magnitude of mode $\sigma$ in substrate be $c_{\sigma}^{(0)}(\mathbf{q})$. The differential intensity of the phonons transmitted into the substrate as a function of $(\omega, t, \mathbf{q})$ and mode $\sigma$, is then ${ }^{7}$ :

$$
i_{\sigma}^{(0)}(\omega, t, \mathbf{q})=A^{(1)} \frac{\hbar \omega^{3}}{8 \pi^{3}} \cdot \frac{\mathbf{e}_{3} \cdot \mathbf{w}_{\sigma}^{(0)}(\mathbf{q})}{\left\{c_{\sigma}^{(0)}(\mathbf{q})\right\}^{3}} \cdot\left\{\frac{1}{e^{\hbar \omega / k_{B} T^{(1)}(t)}-1}-\frac{1}{e^{\hbar \omega / k_{B} T^{(0)}}-1}\right\} \cdot \sum_{\sigma^{\prime}=1}^{3} t_{\sigma \sigma^{\prime}}^{(01)}(-\mathbf{q}) .
$$

Here the differential transmittivity $\frac{8}{\sigma 01)} t_{\sigma \sigma^{\prime}}^{(0)}(-\mathbf{q})$ is used, with the sign of $\mathbf{q}$ inverted, because it should be calculated in the opposite direction assuming the wave is coming from the crystal and transmitted to the radiator.

\subsection{Phonon transportation in the substrate}

A phonon quantum with frequency $\omega$ travels in the direction of its group velocity, carrying an energy of $\hbar \omega$. According to the polarization vector, phonons belong to one of longitudinal (L), fast transverse (FT) and slow transverse (ST) modes 9 . The propagation law of acoustic waves are governed by the elastic constants $C$ and the density $\rho$ of the crystal. Written in component form, the group velocity $\mathbf{w}$ are related with the wave vector $\mathbf{q}$ as: $w_{n}=(1 / \rho \omega) C_{i j l n} e_{i} q_{j} e_{l}$. The normalized polarization vector $\mathbf{e}$ is determined by the wave equation $\rho \omega^{2} e_{i}=C_{i j l m} q_{j} q_{m} e_{l}$.

Mass defects in the lattice lead to scattering of phonons. For a certain type of atom impurity $a$ with mass difference $\Delta m_{a}$ compared to the average atomic mass, the molecule mass $m$, the probability of scattering per unit time to a final mode $f$ within a solid angle range $\Delta \Omega_{f}$ in wave vector $\mathbf{q}$ space is $\frac{10}{}$.

$$
\Gamma_{a}=\frac{1}{16 \pi^{2}} \frac{m}{\rho} f_{a}\left(\frac{\Delta m_{a}}{m}\right)^{2} \omega^{4}\left(\mathbf{e}_{i} \cdot \mathbf{e}_{f}\right)^{2}\left(\Delta \Omega_{f} / c_{f}^{3}\right)
$$

Here $f_{a}$ is number density concentration, calculated as the numbers of impurity atoms $a$ divided by the total number of molecules. $\mathbf{e}_{i}$ and $\mathbf{e}_{f}$ are the polarization vectors of the initial and final states. $c_{f}$ is the phase velocity of the final state. For several impurities, total scattering rate is obtained by summing all impurity type $a$ and solid angles: $\Gamma=\sum_{a} \int d \Omega_{f} \Gamma_{a}$. Let $w$ be group velocity magnitude of the initial phonon, then mean free path is $\lambda=w / \Gamma$. 
On the substrate-vacuum boundary, one phonon quantum reflects with certain probabilities to one of the three modes. For a boundary surface with the normal at direction $\mathbf{z}$ pointing into the crystal medium and an incident with wavevector $\mathbf{q}$, the wavevector component parallel to the surface is $\mathbf{q}_{\|}=\mathbf{q}-(\mathbf{q} \cdot \mathbf{z})$. The Snell's law requires $\mathbf{q}_{\|}$to be equal for incident and reflected waves. In general, there are three reflected wave modes for one incident wave.

The amplitudes for specular reflection waves are related to the incident waves by requiring the vanishing of the stress vector across the surface ${ }^{11}$, because it is a reflection at a stress-free boundary, with the other side being vacuum. While the diffusive reflection is possible, it is not important in experimental setups discussed in this paper and is not considered in physics modeling.

\subsection{Phonon absorption in the sensor}

The incoming phonons which hit the sensor after transportation are not in equilibrium, so each one needs to be treated individually. The total energy deposit of a phonon to the sensor can be calculated as a process of consecutive energy loss of phonon interacting with electrons in a thick medium 12 .

The transmission probability $t_{\sigma}^{(02)}$ for normal incidence and mode $\sigma$ depends on the mass densities $\rho^{()}$and phase velocities $c^{()}$for the substrate (0) and sensor (2), as:

$$
t_{\sigma}^{(02)}=1-\left[\frac{\rho^{(0)} c_{\sigma}^{(0)}-\rho^{(2)} c_{\sigma}^{(2)}}{\rho^{(0)} c_{\sigma}^{(0)}+\rho^{(2)} c_{\sigma}^{(2)}}\right]^{2} .
$$

In addition to the initial transmission, a phonon entering the sensor undergoes attenuation in the metal and multiple reflections along the interfaces. As a result, the total energy deposit is the incident phonon energy scaled by an absorption coefficient for sensor material 13 .

$$
a_{\sigma}^{(02)}(\omega)=t_{\sigma}^{(02)} \cdot \frac{1-e^{-4 \alpha_{\sigma}^{(2)}(\omega) l^{(2)}}}{1-\left(1-t_{\sigma}^{(02)}\right) e^{-4 \alpha_{\sigma}^{(2)}(\omega) l^{(2)}}}
$$

Here the absorption constant $\alpha_{\sigma}^{(2)}(\omega)=\alpha_{\sigma}^{(2)}\left(\omega_{0}\right) \cdot \omega / \omega_{0}$ for mode $\sigma$ is proportional to the phonon frequency $\omega . l^{(2)}$ is the sensor film thickness.

By accumulating large amount phonons hitting at sensor at different times, the input power as a function of time is obtained. Using Eq. 2 by replacing the material superscript (1) to (2), the temperature rise as a function of time can be subsequently calculated.

\section{Simulation Procedure}

The actual simulation follows the physics model presented at the previous sections. Phonon tracks are monitored from the creation until its absorption in the sensor using modern Monte Carlo techniques. The detailed procedures for one phonon track are:

- Generate one phonon track traveling into the halfspace of crystal. The initial vertex is evenly distributed on the radiator-crystal interface area. The mode, direction and energy follow the distribution in Eq. 3 Here the base temperature $T^{(0)}$ for substrate follows experimental condition, and the temperature for the radiator $T^{(1)}$ is obtained from Eq. 2 . assuming steady condition with a constant input power $P^{(10)}$ 
- Propagate the phonon track inside the crystal substrate. The propagation is simulated with many connected tracks, with the direction following its group velocity. The length of one individual track is determined by sampling the exponential distribution with the mean free path as its mean value. The scattering process is implemented by generating a new track, with the direction in q space and mode $f$ distributed in Eq. 4. If the length of a track runs beyond the boundary of the substrate, then it is reflected. The mode of reflection is generated with a probability proportional to the power transmission across the interface. If the track hits the area of the sensor, it is removed from the simulation and the energy deposit is calculated as in the absorption process of the next step.

- Phonon absorption. When a phonon track hits the boundary between the sensor and the crystal, the energy deposit to the sensor is calculated as the phonon energy multiplied by the absorption coefficient $a_{\sigma}^{(02)}(\omega)$ in Eq. 6

The starting time of a phonon track from the radiator is uniformly sampled in the window of the heater pulse width. To proceed a full simulation, huge number of phonon tracks are generated and propagated, such that we can obtain a smooth distribution of energy deposit versus time for the sensor. If absolute energy deposit or temperature rise in the sensor is needed, the absolute $y$-axis value is scaled according to the ratio of total generated phonon energy over total input energy during the input pulse.

\section{Experiments}

A simulation can be claimed useful only if it can explain and predict the experimental data. We use two experimental setups: A as in Ref. ${ }^{14}$ and B as in Ref. ${ }^{[15}$ to perform the simulation. The input conditions are exactly the same as in the referenced papers. For setup A, the material for radiator, substrate, and sensor are aluminum, $m$-cut sapphire crystal and tin. For setup B, the corresponding materials are constantan, [111]-cut silicon, and aluminum. The geometries for two setups are illustrated in Fig. 1. The thickness of sensor are 200 (50) nm for setup A (B). The base temperatures are $2.0 \mathrm{~K}$ for setup A, and $1.369 \mathrm{~K}$ or $1.36 \mathrm{~K}$ for two experiments in setup B.

The surface with radiator and sensor film in setup A is immersed in liquid helium instead of vacuum. The effect of helium contact to radiator and sensor is considered by assuming that $50 \%$ of phonons ${ }^{16}$ travel from the metal to the liquid helium at the interface, which largely gives an overall scale change to the total emission and absorption powers. Because the experimental result of Setup A is a plot with arbitrary unit in signal height, this effect is not relevant. The reflection surface in setup A is kept in vacuum or liquid helium. From the study in Ref. ${ }^{14}$, if the reflecting surface is in contact with liquid helium, no energy leak will happen for the specular reflection, while the diffusely scattered phonons are lost due to the anomalous Kapitza effect. Since the diffusive scattering process are not considered in our simulation, we shall use the experimental data with reflection surface in liquid helium in comparison (the dashed line in the top plot of Fig. 2).

In the simulation, the elastic constants of the crystals are obtained from Ref. 17 for sapphire and Ref. ${ }^{15}$ for silicon. The radiator's elastic constants are calculated from the longitudinal and transverse velocities in Ref. ${ }^{5}$. The absorption coefficient $\alpha_{\sigma}^{(2)}(100 \mathrm{GHz})$ used for the aluminum and tin metal sensor are measured values, as in Table I of Ref. 13 .

A total of 100 million phonons are generated for setup A. And for each experimental conditions in setup B, 40 million phonons are generated. The simulated timing spectrum of the energy deposit or temperature rise in the sensor are shown and compared to the experimental data in Figs. 2 and 3 . The time windows are chosen according to the published plots 

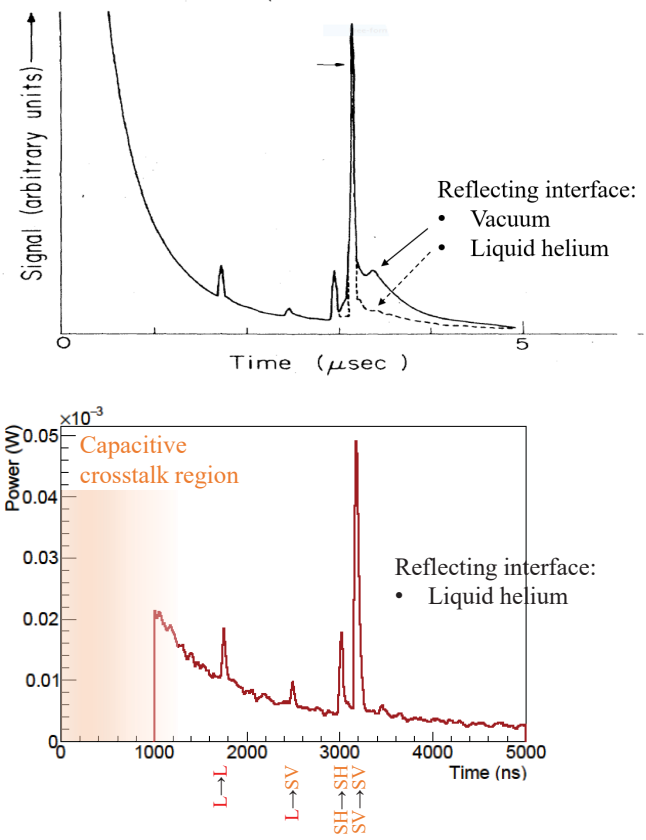

Fig. 2 Spectra of energy deposit for Experimental setup A with a heater pulse width of $35 \mathrm{~ns}$. The top plot is for real experimental data from Taborek and Goodstein 14 and the bottom plot is for the simulation result. The simulation is under a condition with the reflection surface in contact with liquid helium, and should be compared with dashed line in the top plot.

of real data. All plots show one or several peaks due to reflection lying on a broad shape due to scattering.

In Fig. 2 for setup A, the time window is wide enough to see various peaks. Since the radiator-sensor is in the $C-Y$ plane, the transverse modes can also be labeled as shear horizontal $(\mathrm{SH})$ and shear vertical $(\mathrm{SV})$ too. The four distinct peaks with various intensity correspond to the reflections of $\mathrm{L} \rightarrow \mathrm{L}, \mathrm{L} \rightarrow \mathrm{SV}, \mathrm{SH} \rightarrow \mathrm{SH}$ and $\mathrm{SV} \rightarrow \mathrm{SV}$ transitions. Other possible reflections are not seen due to limited wave vector phase space or low reflection probabilities.

For setup B, in the left column of Fig. 3 three experimental conditions are applied with different heater pulse power, resulting the temperature of the radiator being 5.25, 5.56 and 5.89 Kelvin. The width of time window is set to be just beyond the first clear $\mathrm{L} \rightarrow \mathrm{L}$ reflection peak at around $1450 \mathrm{~ns}$. Broad bumps before the reflection peaks are due to bulk scattering, which have maxima at around $600 \mathrm{~ns}$ from geometry effect of scattering vertices. In the right column of Fig. 3. superimposed plots are normalized to same maximum height, with five different experiments with various radiator temperatures. Here the relative size of reflection peaks decreases as the radiator temperature rises, which is due to the scattering probability being proportional to the fourth power of the energy of individual phonons. As phonon energy increases, it will encounter more scattering against ballistic reflection.

We see an excellent agreement between the simulation and real data in Fig. 2 for setup A, and in left and right columns of Fig. 3 for three and five different setup B conditions 

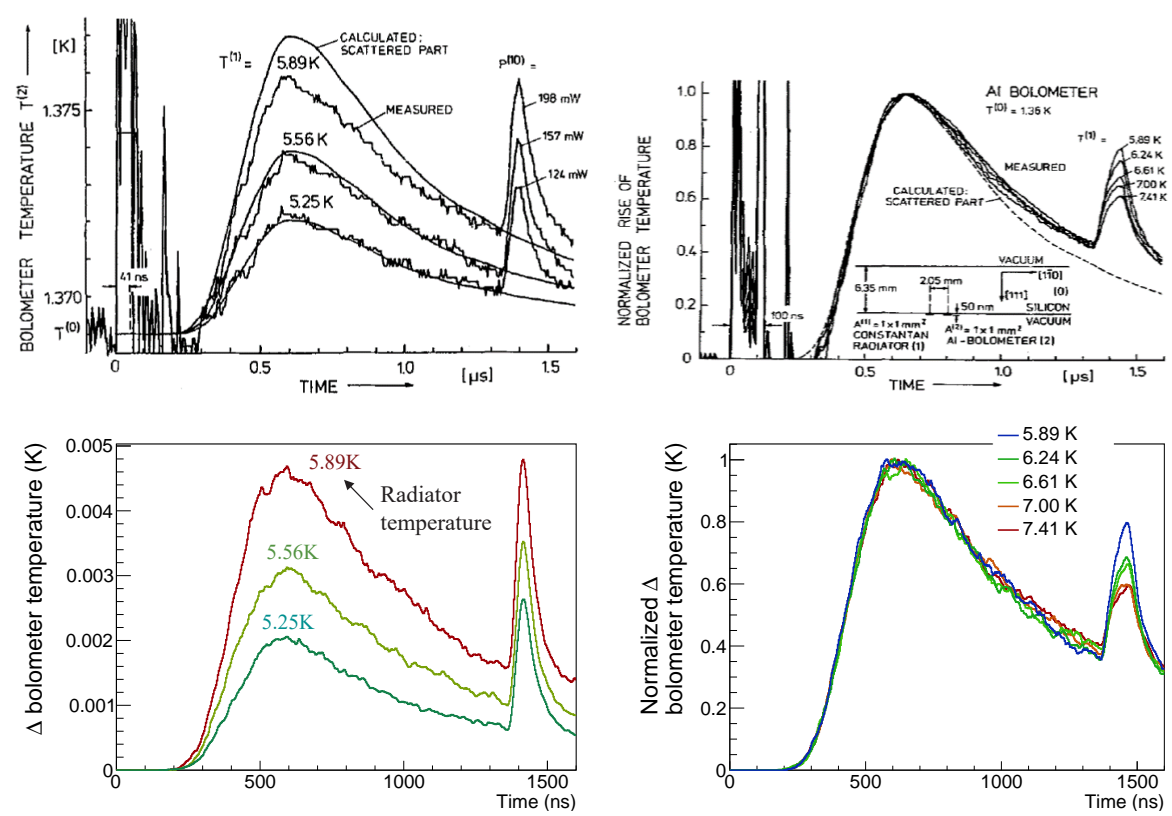

Fig. 3 Spectra of temperature rise for setup B. The heater pulse width is $41 \mathrm{~ns}$ (left) and $100 \mathrm{~ns}$ (right). The spectra labeled as "MEASURED" in the top row is for real experimental data from Mrzyglod and Weis 15 and the bottom row is for the simulation result. Results with different radiator temperatures are plotted together. The right column shows temperature rise normalized to same maximum height.

respectively. This validates our model, at least for the metal heater in a substrate geometry, where the initial heat pulse arrivals to the sensor with reflection.

Previous attempts in Refs. $\frac{187719}{7}$ have tried to explain the observed signal shape and magnitude. Those methods used numerical iteration to determine all possible phonon reflection paths, and numerical or analytical integration over all the scattered phonon travel paths, by treating the radiator and sensor as single points. They are limited to time range of the first reflection, single scattering in weak scattering limit, small size radiator and sensor, thin geometry and can not extend to other applications straightforwardly. Moreover, in Ref. 18 , a quantitative explanation of the size of each peak cannot be obtained for the multiple-peak spectrum in Setup A. In a simulation reported on Ref. ${ }^{2}$, no reflection process is considered, no full anisotropic treatment of scattering is done, and no rigorous check has been made for different experimental conditions. On the contrary, with the full simulation method presented in this work, none of the above limits exist. In addition, we are free to extend with other physical processes which may be important in different experimental conditions. It is clear that simulation methods similar to our model must be done to explain the experimental data quantitatively in real applications.

The next step is to apply our model to larger detectors and longer collection time. Examples are cryogenic crystal detectors for rare event search experiments, such as $\mathrm{CaMoO}_{4}$ based neutrinoless double beta decay search $\mathrm{AMoRE}^{20}$ and $\mathrm{CaWO}_{4}$ based dark matter search CRESST ${ }^{21}$. It is of importance to understand phonon creation process by energy deposit from elementary particles. Also, additional phonon propagation process may need to be considered, for instance, the diffusive scattering at the boundary, which can affect the energy transportation at longer time scale. 


\section{Conclusion and Discussion}

A new model has connected the real signals of low temperature crystal and the underlying first principle physical processes. It is a result of combining the evolving computing technology and the basic theory of acoustic waves. Thus, from now, our community has a new tool to explain and predict full series of data spectrum quantitatively. This tool is expected to be useful when applying to larger and more complex experimental setups. Here, new questions in phonon physics may appear in the development of phonon-based detectors.

Acknowledgements The author would like to thank Yonghamb Kim, Juhee Lee, Chang Lee and Seungyoon Oh for useful communications. This work was supported by project code IBS-R016-D1.

\section{References}

1. H. J. Maris, J. Low Temp. Phys. 93, 355, (1993).

2. D. Brandt, M. Asai, P.L. Brink et al., J. Low. Temp. Phys. 167, 485, (2012).

3. Steven W. Leman, Review of Scientific Instruments 83, 091101, (2012).

4. O. Weis, Z. Angew. Phys. 26, 325, (1969).

5. F. Rösch and O. Weis, Z. Phys. B 27, 33, (1977).

6. R. Bayrle and O. Weis, J. Low Temp. Phys. 76, 129, (1989).

7. G. Müller and O. Weis, Z. Phys. B 80, 25, (1990).

8. O. Weis, Z. Phys. B 34, 55, (1979).

9. J.P. Wolfe, Imaging Phonons, Cambridge Univ. Press, Cambridge, 1998.

10. O. Weis, Z. Phys. B 96, 525, (1995).

11. F. I. Fedorov, Theory of Elastic Waves in Crystals, Ch. 8, Plenum, New York, 1968.

12. M. Goetze, M. Nover and O. Weis, Z. Phys. B 25, 1, (1976).

13. A. Mrzyglod and O. Weis, J. Low Temp. Phys. 97, 275, (1994).

14. P. Taborek and D. Goodstein, Phys. Rev. B 22, 1550, (1980).

15. A. Mrzyglod and O. Weis, Z. Phys. B 97, 103-112, (1995).

16. T. L. Head and M. E. Msall, Chinese Journal of Physics 49, 278, (2011).

17. A. G. Every and G. L. Koos and J. P. Wolfe, Phys. Rev. B 29, 2190, (1984).

18. P. Taborek and D. Goodstein, J. Phys. C 12, 4737, (1979).

19. G. Müller and O. Weis, Z. Phys. B 80, 15, (1990).

20. G. B. Kim, S. Choi, F. A. Danevich, et al., Advances in High Energy Physics, vol. 2015, Article ID 817530, 7 pages, 2015.

21. G. Angloher et al. [CRESST Collaboration], Eur. Phys. J. C 76, no. 1, 25 (2016). 